\title{
Von der sozialen Neuzusammensetzung zur gewerkschaftlichen Erneuerung? MigrantInnen als Zielgruppe der österreichischen Gewerkschaftsbewegung
}

\author{
Markus Griesser • Birgit Sauer
}

(C) Der/die Autor(en) 2017. Dieser Artikel ist eine Open-Access-Publikation.

Zusammenfassung Der Beitrag fragt danach, wie sich österreichische Gewerkschaften und Arbeiterkammern beim Versuch, MigrantInnen als Zielgruppe zu erreichen, verändern. Diese Fragestellung wird ausgehend von konkreten Einrichtungen der solidarischen Beratungs- und Unterstützungsarbeit behandelt, wie sie von ArbeitnehmerInnenorganisationen mit Blick auf die besonderen Interessen und Bedürfnisse von MigrantInnen etabliert wurden. Vor dem Hintergrund der gewerkschaftlichen Mehrfachkrise setzen diese bislang vornehmlich auf traditionelle Dienstleistungsstrategien, um migrantische Beschäftigte als Mitglieder zu gewinnen. Aus der Perspektive eines Machtressourcenansatzes wird jedoch vorgeschlagen, solche Praxen als Bestandteile einer umfassenden gewerkschaftlichen Erneuerung zu begreifen, die den Einsatz für die Stärkung der Organisationsmacht von MigrantInnen zu einer zentralen Grundlage der organisationseigenen Stärke von Gewerkschaften macht.

Schlüsselwörter Gewerkschaften · Migration · Arbeitsmärkte $\cdot$ Machtressourcen

\section{From Social Recomposition to Union Revitalization? Migrants as a target group of the Austrian Trade Union Movement}

\footnotetext{
Abstract The article deals with the question how organised labour in Austria has to change in order to successfully address migrant workers. To answer this research question we focus on supportive practices developed by Austrian trade unions and chambers of labour with respect to the specific needs and interests of migrants.

M. Griesser $(\bowtie) \cdot$ B. Sauer

Institut für Politikwissenschaft, Universität Wien, Universitätsstraße 7/2. Stock, 1010 Wien,

Österreich

E-Mail: markus.griesser@univie.ac.at

B. Sauer

E-Mail: birgit.sauer@univie.ac.at
} 
The article argues that these supportive practices have to be assessed on one hand against the background of the multiple crises of the trade union movement. Hence, we identified strategies predominantly associated with the traditional service model, which aims at organizing migrant workers. On the other hand, from the perspective of the power resource approach those supportive practices can be seen as part of a broader strategy of union revitalization, which is aiming at consolidating the strength of trade unions by strengthening the associational power of migrant workers.

Keywords Trade unions · Migration - Labour markets · Power resources

\section{Einleitung 1}

Was das neo-korporatistische Arrangement der Interessenvermittlung und seine tragenden Säulen anbelangt, galt im Österreich der Zweiten Republik lange Zeit in historisch wie international vergleichender Perspektive ein hohes Maß an Stabilität als zentrales Charakteristikum. Dieses wurde mit dem Fortbestehen der stark zentralisierten und vereinheitlichten Interessenverbände von Arbeit und Kapital begründet, aber auch mit der anhaltenden Relevanz „sozialpartnerschaftlicher“ Muster der Interessenkonzertierung und -akkordierung im Bereich der Einkommensund Lohnpolitik ebenso wie in anderen sozial- und wirtschaftspolitischen Feldern (vgl. etwa Ebbinghaus 2000; Traxler 1998). Durch diese Diagnose relativer Stabilität wurde jedoch weder ein gradueller Bedeutungsverlust (vgl. Karlhofer 2012) noch ein sukzessiver Formwandel (vgl. Traxler 1993) neo-korporatistischer Gestaltungsmacht, die in den 1980er-Jahren einsetzten und ab Mitte der 1990er-Jahre an Intensität gewannen, infrage gestellt. Verantwortlich für diese Veränderungen waren ökonomische Faktoren wie steigende Erwerbsarbeitslosigkeit, fortschreitende Tertiarisierung oder verschärfter Wettbewerbsdruck im Zuge der neoliberalen Globalisierung, aber auch politische Faktoren wie der europäische Integrationsprozess oder die gewerkschaftsfeindliche Politik der ÖVP/FPÖ/BZÖ-Regierung Anfang der 2000er-Jahre (vgl. etwa Gstöttner-Hofer 2005, S. 29 ff.; Tálos 2008, S. 77 ff.).

Neben dem neo-korporatistischen Arrangement durchlief mit dem Österreichischen Gewerkschaftsbund (ÖGB) und seinen Teilgewerkschaften aber auch eine seiner tragenden Säulen einen Prozess krisenhafter Transformation, wurde diese doch ab den 1980er-Jahren in fortschreitendem Umfang von der (zumindest) ,,,dreifache[n] Krise" der Gewerkschaften“ (vgl. etwa Holst et al. 2008) erfasst. Diese Krise artikuliert sich hierzulande nicht primär als Legitimitäts-, sondern als Mitgliederund Finanzkrise (vgl. ebd., S. 161; Pernicka und Stern 2011, S. 336). ${ }^{2}$

\footnotetext{
1 Vorarbeiten für diesen Artikel wurden im Rahmen zweier Forschungsprojekte (Organised Labour and Migrant Workers, 02-10/2013 sowie MigrantInnen als Zielgruppe, 03-09/2014) am Institut für Politikwissenschaft der Universität Wien durchgeführt und seitens der Europäischen Kommission (Agreement ref. no. VS/2012/0425) sowie der Arbeiterkammer Wien gefördert. Für ihre Arbeit und hilfreiche Anmerkungen danken wir den HerausgeberInnen und anonymen GutachterInnen des vorliegenden Themenhefts der ÖZS.

2 Dass die Kammer für Arbeiter und Angestellte (AK) als gesetzliche Interessenvertretung der abhängig Beschäftigten in Österreich von dieser Krise - zumindest was die Frage des Organisierungsgrads (Pflicht-
} 
Was den zweitgenannten Aspekt anbelangt, verlor der ÖGB hinsichtlich seiner Organisationsmacht zunehmend an Boden. Vor dem Hintergrund weitreichender Veränderungen des Arbeitsmarkts und grundlegender Neuzusammensetzungen der Beschäftigten ist in den vergangenen Jahrzehnten eine sukzessive Ausdünnung seiner Mitgliederbasis zu beobachten. War die unmittelbare Nachkriegszeit noch durch einen im internationalen Vergleich hohen gewerkschaftlichen Organisationsgrad gekennzeichnet, veränderte sich dies ab den 1960er-Jahren langsam und ab den 1980erJahren mit zunehmender Geschwindigkeit (vgl. Blaschke 2007, S. 247 f.; Traxler und Pernicka 2007, S. $212 \mathrm{ff}$.): Laut Angaben der Organisation für wirtschaftliche Zusammenarbeit und Entwicklung (OECD 2016) sank der Organisationsgrad in Österreich zwischen 1960 und 2013 von 67,9\% auf 27,8\%. Mit dem 2006 publik gewordenen Skandal um die ÖGB-eigene Bank für Arbeit und Wirtschaft AG (BAWAG), die infolge riskanter Spekulationsgeschäfte massive Verluste eingefahren hatte, spitzte sich kurzzeitig nicht bloß die Mitgliederkrise des ÖGB weiter zu, sondern - was langfristig von noch größerer Relevanz scheint - auch seine Finanzund partielle Legitimitätskrise (vgl. Karlhofer 2012, S. 531 f.). Verschärft wird dieses Problem zum einen durch eine ,wachsende Vertretungslücke auf betrieblicher Ebene“ (Hermann und Flecker 2009, S. 93), die aus dem Umstand einer rückläufigen Zahl von Betriebsratskörperschaften resultiert, und zum anderen durch die Schwächung der traditionell engen Beziehung zwischen Betriebsratskörperschaften und Gewerkschaften etwa aufgrund der steigenden Anzahl nicht-gewerkschaftlich organisierter BetriebsrätInnen (vgl. Tálos 2008, S. 90 ff.).

In der Gewerkschaftsforschung führten diese Entwicklungen mitunter dazu, für Österreich eine „Erosion gewerkschaftlicher Organisations- und Durchsetzungsmacht“ (Brinkmann et al. 2008, S. 51) zu konstatieren, die durch den einseitigen Fokus auf - vermeintliche oder tatsächliche - institutionelle Stabilitäten verdeckt werde. Zugleich wurde das fortschreitende Schwinden der Organisationsmacht zum Auslöser verschiedener Initiativen einer gewerkschaftlichen Erneuerung (vgl. etwa Blaschke 2002, 2007; Gstöttner-Hofer 2005, S. 93 ff.; Pernicka und Stern 2011, S. 336 ff.). In diesem Kontext gewannen neben Frauen auch MigrantInnen ${ }^{3}-$ also jene Gruppen, die unter den Beschäftigten der expandierenden Dienstleistungssektoren überrepräsentiert sind - als Zielgruppen der Gewerkschaften an Bedeutung (vgl. Biffl 2011, S. 132 f./138).

Durch diese Entwicklungen wurden migrationspolitische Dilemmata, mit denen ArbeitnehmerInnenorganisationen nicht nur hierzulande bereits seit langem konfrontiert sind (vgl. etwa Penninx und Roosblad 2000; Fine und Tichenor 2012), in neuer Form aktualisiert. Hier setzt der vorliegende Artikel an, indem er danach fragt,

\footnotetext{
mitgliedschaft) und der Finanzierung (Arbeiterkammerumlage) betrifft - nicht oder nicht in gleicher Form betroffen ist, sei hinzugefügt. Und auch die Ende der 1980er- bzw. Anfang der 1990er-Jahre evidente „Kammerkrise“ (Karlhofer 2006, S. 471 f.) scheint - soweit es sich dabei um eine Legitimitätskrise handelte - vorerst überwunden.

3 Auch wenn wir uns der Schwächen eines solch weiten Begriffsverständnisses bewusst sind, verwenden wir den Terminus ,MigrantInnen“ im vorliegenden Artikel in einem übergreifenden Sinn für Personen mit Migrationserfahrungen. Fragen der Staatsangehörigkeit oder des Aufenthaltstitels bleiben dabei insofern unberücksichtigt, als diese für den hier behandelten Gegenstand - gewerkschaftliche Beratungs- und Unterstützungsangebote für MigrantInnen - von nachrangiger Bedeutung sind.
} 
wie sich österreichische Gewerkschaften und Arbeiterkammern vor dem skizzierten Hintergrund beim Versuch, MigrantInnen zwecks Stärkung ihrer Organisationsmacht als (neue) Zielgruppe zu erreichen, verändern. Der Fokus liegt auf Kriterien einer gelungenen solidarischen Beratungs- und Unterstützungsarbeit, wie sie in diesem Rahmen entwickelt wurde. Um die dargelegte Fragestellung zu beantworten und die erwähnten Gütekriterien herauszuarbeiten, untersuchten wir konkrete Einrichtungen, die von österreichischen ArbeitnehmerInnenorganisationen mit Blick auf die besonderen Interessen und Bedürfnisse von MigrantInnen etabliert wurden. Dazu skizzieren wir zunächst den theoretischen Rahmen der Untersuchung (Abschn. 2) und anschließend die gewerkschaftlichen Beratungs- und Unterstützungseinrichtungen (Abschn. 3), bevor in der Folge die Beantwortung der Forschungsfrage ins Zentrum rückt (Abschn. 4). Abschließend wird der Argumentationsgang resümiert, und wir präsentieren einige Schlussfolgerungen für eine strategisch erneuerte Gewerkschaftsarbeit im Kontext der österreichischen Migrationsgesellschaft (Abschn 5).

\section{Gewerkschaften und Migration: theoretische Ausgangsüberlegungen}

Vor dem Hintergrund ihrer Mehrfachkrise stellt sich die Frage der „Organisierbarkeit“" von MigrantInnen für österreichische Gewerkschaften heute in vielen Branchen nicht mehr bloß als eine der Solidarität, sondern - zugespitzt formuliert - immer mehr auch als eine ihres eigenen Überlebens (vgl. ähnlich für Deutschland bzw. Großbritannien Artus 2011, S. 216; Fitzgerald und Hardy 2010, S. 145). Dies ergibt sich bereits aus dem schlichten Umstand, dass die Zahl der unselbstständig Erwerbstätigen ohne österreichische Staatsbürgerschaft in Österreich seit 1998 (mit Ausnahme des „Krisenjahres“ 2009) kontinuierlich von damals rd. 298.600 Personen bzw. 10\% auf rd. 615.700 Personen bzw. 17,4\% im Jahr 2015 anwuchs (vgl. Biffl 2016, S. 92 ff.). ${ }^{4}$

Im Besonderen gilt dies für Branchen, die einen hohen MigrantInnenanteil aufweisen, namentlich etwa für die Land- und Forstwirtschaft (z. B. Erntehilfe), die Beherbergung und Gastronomie (z. B. Tourismus), die Unternehmensdienstleistungen (z. B. Gebäudereinigung), die privaten Haushalte (z. B. Pflegearbeit) und das Bauwesen (z. B. Bauarbeit), wo im Juni 2015 zwischen 25,1\% (Bauwesen) und $58,2 \%$ (Land- und Forstwirtschaft) der hier jeweils Beschäftigten ohne österreichische Staatsbürgerschaft waren (vgl. Biffl 2016, S. 100 ff.). Keineswegs zufällig ist es in diesen Branchen - und speziell in jenen Teilbereichen, die als ,ethnisch segmentierte Arbeitsmärkte" charakterisiert werden können (Krenn et al. 2012, S. 33 ff./91 f.; vgl. auch Riesenfelder et al. 2011, S. 52 ff.) - um die Entgelt- und Arbeitsbedingungen vielfach besonders schlecht bestellt und werden sozial- und arbeitsrechtliche Standards regelmäßig unterlaufen. Zudem gibt es starke Überschnei-

\footnotetext{
${ }^{4}$ Noch deutlicher tritt der Wandel in langfristiger Perspektive zu Tage, lagen die entsprechenden Zahlen doch etwa im Jahr 1961, also zu Beginn der Anwerbephase von sogenannten „GastarbeiterInnen“, noch bei rd. 16.200 Personen bzw. 0,7\%. Hinzu kommt, dass Migrationsprozesse nicht bloß quantitativ an Bedeutung gewannen, sondern sich auch qualitativ veränderten (z. B. durch die Zunahme temporärer gegenüber dauerhaften Formen der Migration).
} 
dungen mit solchen Branchen, in denen - im Sinne eines ,shadowing effect“" (Krenn und Haidinger 2009, S. 23) informeller auf formelle migrantische Beschäftigung auch un(ter)dokumentierte Arbeit von MigrantInnen weit verbreitet ist (vgl. auch Jandl et al. 2007, S. 34 ff.).

Aus der Perspektive des Machtressourcenansatzes verweist die prekäre Situation in den genannten Branchen zentral auf die mangelhafte Ausstattung der hier Beschäftigten mit strukturellen und institutionellen Machtressourcen. Den theoretischen Ausgangspunkt des Ansatzes bildet die Annahme, dass das Verhältnis zwischen Arbeit und Kapital zwar aufgrund der bestehenden Eigentums- und Besitzverhältnisse durch eine grundlegende Asymmetrie gekennzeichnet ist, dass diese jedoch in Abhängigkeit von der Mobilisierung unterschiedlicher Machtressourcen variiert. Von zentraler Bedeutung ist dabei die Bildung von „Organisationen kollektiven Handelns“ wie etwa Gewerkschaften, die es erlauben, die Machtressourcen verschiedener Individuen bzw. Gruppen zu koordinieren und darüber deren relative Unterlegenheit zu kompensieren (vgl. etwa Korpi 1983, S. 14 ff.; Olsen und O’Connor 1998, S. 6).

Der Jenaer Arbeitskreis Strategic Unionism machte den Machtressourcenansatz für die Gewerkschaftsforschung produktiv (vgl. einführend Brinkmann et al. 2008, S. $24 \mathrm{ff}$, und der Artikel von Dörre in diesem Heft): ${ }^{5}$ Unter Bezugnahme auf Beverly J. Silver (2005, S. 30 ff.) und Eric Olin Wright (2000, S. 962), die Macht als individuelle oder kollektive Fähigkeit zur Durchsetzung von (Klassen-)Interessen begreifen, unterscheiden die Jenaer ForscherInnen in einem ersten Schritt zwischen struktureller Macht (structural power), die aus der Stellung der Arbeitenden im Wirtschaftssystem resultiert, und Organisationsmacht (associational power), die aus ihrer Vereinigung etwa zu Gewerkschaften folgt. Im Bereich der strukturellen Macht differenzieren sie wiederum zwischen Produktionsmacht (workplace bargaining power), die von der strategischen Positionierung im Produktionsprozess abhängt (z. B. Störpotenzial durch Streik), und Marktmacht (marketplace bargaining power), die mit der Stellung am Arbeitsmarkt verbunden ist (z. B. Drohpotenzial aufgrund seltener Qualifikationen). In einem zweiten Schritt erweitern sie das Modell von Wright und Silver um eine dritte grundlegende Form, nämlich die institutionelle Macht, die aus institutionell sedimentierten und häufig gesetzlich festgeschriebenen Basiskompromissen resultiert.

Was nun die oben genannten Branchen anbelangt - von der Land- und Forstwirtschaft bis zum Bauwesen, von den unternehmensorientierten bis zu den personennahen Dienstleistungen -, so scheinen diese dadurch gekennzeichnet, dass die Ausstattung der hier Beschäftigten mit strukturellen und institutionellen Machtressourcen in der Regel schwach ausgeprägt ist. Gerade MigrantInnen finden sich

\footnotetext{
5 Das im Folgenden dargestellte Grundmodell des Jenaer Machtressourcenansatzes wurde in späteren Arbeiten auf vielfältige Art und Weise fortentwickelt (für einen Überblick vgl. Arbeitskreis Strategic Unionism 2013). Auf den Ansatz Bezug genommen wird hier - wie auch in anderen neueren Arbeiten zum Thema im deutschsprachigen Raum (vgl. etwa Artus 2011; Benz 2014; Schröder 2015) - vor allem deshalb, weil er eine gesellschaftstheoretisch fundierte Auseinandersetzung mit dem Verhältnis von Gewerkschaften und Migration ermöglicht und es durch seinen spezifischen Fokus auf Machtressourcen erlaubt, unterschiedliche Dimensionen dieses Verhältnisses zu differenzieren und deren Bedeutung für Fragen der gewerkschaftlichen Erneuerung herauszuarbeiten.
} 
hier nämlich vielfach auf sogenannte ,Jedermannsteilarbeitsmärkte“ (Sengenberger 1978, S. 61 ff.; Kalter 2005, S. 311 ff.) konzentriert, die aufgrund unspezifischer Qualifikationsanforderungen und entsprechend leichter Substituierbarkeit mit wenig Marktmacht sowie aufgrund ihrer marginalen Stellung im Produktionsprozess und in der gesellschaftlichen Arbeitsteilung mit ebenso wenig Produktionsmacht verbunden sind. ${ }^{6}$ Hinzu kommt, dass unter anderem infolge der dargestellten „Vertretungslücke“ auf betrieblicher Ebene in diesen vielfach durch räumliche Fragmentierung gekennzeichneten Branchen (z. B. kleinbetriebliche Strukturen, Subunternehmertum) auch institutionelle Machtressourcen häufig eine geringere Rolle spielen.

Wegen der wirtschaftlichen Bedeutung vor allem von Branchen wie den unternehmensorientierten bzw. den personennahen Dienstleistungen sowie aufgrund des Mangels an strukturellen und institutionellen Machtressourcen aufseiten der hier Beschäftigten erhält die Frage einer strategisch erneuerten Organisationsmacht wachsende Relevanz für aktuelle ArbeiterInnen- und Gewerkschaftsbewegungen (vgl. Silver 2005, S. 134 ff./214 ff.; Artus 2011, S. 209 ff.). Nicht von ungefähr markieren im Rahmen der internationalen Labor Revitalization Studies (LRS) die Stärke und Zusammensetzung der Mitgliederbasis von Gewerkschaften eine zentrale Dimension für die Erneuerung ihrer Handlungs- und Konfliktfähigkeit (vgl. etwa Voss und Sherman 2000, S. 310 ff./316; Behrens et al. 2004, S. 20 f.). Wenngleich die Richtung, der Einsatz und die Methoden der damit verbundenen Bemühungen divergieren, gewann in diesem Zusammenhang auch die Frage der gewerkschaftlichen Organisierung von bislang nicht bzw. unterrepräsentierten Gruppen wie MigrantInnen an Bedeutung (vgl. Heery und Adler 2004; Milkman und Wong 2001). Oder wie Craig Phelan (2007, S. 26) es formuliert: „Organising those who have been traditionally excluded or underrepresented in trade union movements has become a key component of revitalising strategies."

In einigen Ländern wie etwa in den USA haben Gewerkschaften in diesem Zusammenhang eine grundlegende Neupositionierung zu den eingangs angesprochenen Dilemmata in Bezug auf das Themenfeld Migration bzw. auf MigrantInnen als Zielgruppe vollzogen (vgl. etwa Meyer 2002, S. 119 ff.; Adler und Cornfield 2014). Im Rahmen neuartiger Organisierungsmodelle und -kampagnen konnten so umfangreiche Erfahrungen mit Ansätzen gewonnen werden, die sich speziell an den besonderen Interessen und Bedürfnissen dieser Zielgruppe orientierten (vgl. etwa Choi 2008; Benz 2014). Doch nicht bloß in den USA, auch in europäischen Ländern wie beispielsweise in Frankreich oder Großbritannien gingen Gewerkschaften in den vergangenen Jahrzehnten von einer vornehmlich restriktiven zu einer stärker

\footnotetext{
6 Nicht bloß Silver (2005, S. $69 \mathrm{ff} . / 91 \mathrm{ff}$.) hat darauf verwiesen, dass diese Tendenz einer abnehmenden Markt- und Produktionsmacht in vielen der führenden Branchen der Gegenwart nicht überbetont werden sollte, da es selbst im Bereich der unternehmensorientierten und personennahen Dienstleistungen gegenläufige Tendenzen gibt. So seien zentrale Kapitalstrategien wie etwa ,räumliche fixes“ im Sinn von geografischen Verlagerungen sowie „technologische fixes“ im Sinn von Prozessinnovationen (z. B. Automation) gerade in diesen stark ortsgebundenen und arbeitsintensiven Branchen schlicht nicht anwendbar (vgl. auch Alberti et al. 2014, S. 113). Dies impliziert eine relative Stärkung der Markt- und Produktionsmacht der hier Beschäftigten, was der von Kapitalseite in solchen Branchen entwickelten Strategie der Flexibilisierung und Kostensenkung durch erhöhten Druck auf Löhne und Sozialstandards (vgl. etwa Hardy et al. 2012, S. 359; Meardi et al. 2012, S. 7 ff.) (potenziell) Grenzen setzt.
} 
solidarischen Haltung gegenüber MigrantInnen über und entwickelten spezifische Angebote für diese Zielgruppe (vgl. etwa Fine und Tichenor 2012; Adler et al. 2014). Für ArbeitnehmerInnenorganisationen in den Mitgliedstaaten der Europäischen Union (EU) markierten in diesem Kontext die fortschreitende Transnationalisierung des Arbeitsmarkts und die damit verbundenen Formen einer häufig temporären (OstWest-)Migration eine besondere Herausforderung (vgl. etwa Krings 2009; Hardy et al. 2012; Meardi et al. 2012). Doch auch in Reaktion darauf erweiterten in jüngster Zeit Gewerkschaften in Ländern wie Deutschland (vgl. etwa Greer et al. 2013; Schröder 2015, S. 137 ff.) und Großbritannien (vgl. etwa Fitzgerald und Hardy 2010; Mustchin 2012) auf innovative Weise ihr Strategie- und Handlungsrepertoire.

Für Österreich hingegen konstatierte Sabine Blaschke (2002, S. 97) noch im Jahr 2002 im Kontext der Revitalisierungsdebatte: „Auffällig ist, dass die Gewerkschaften sich um MigrantInnen als spezielle Zielgruppe nicht bemühen" (vgl. ähnlich auch bereits Bauböck 1990, S. 49). Diese Einschätzung erklärt sich vor dem Hintergrund der Ambivalenzen, wie sie für das Verhältnis österreichischer ArbeitnehmerInnenorganisationen zum Themenfeld Migration bzw. zu MigrantInnen als Zielgruppe lange Zeit offensichtlich waren (vgl. exemplarisch Gächter 2000; Kreisky 2013). Erst in jüngster Zeit finden sich in der Literatur vermehrt Hinweise auf einen Wandel dieses Verhältnisses - und damit in Zusammenhang stehend auch Ansätze einer gewerkschaftlichen Praxis, die mittels Dienstleistungs- und/oder Organisierungsstrategien auf MigrantInnen als potenzielle Mitglieder zielen (vgl. etwa Biffl 2016, S. 139).

Vor diesem Hintergrund, der andernorts im Detail rekonstruiert wurde (vgl. Griesser und Sauer 2015), soll im Folgenden der Frage nachgegangen werden, wie sich österreichische ArbeitnehmerInnenorganisationen veränderten, um MigrantInnen als (neue) Zielgruppe zu erreichen. Welche Faktoren sind im Hinblick auf dieses Ziel von Bedeutung, und inwiefern gelingt es österreichischen Gewerkschaften und Arbeiterkammern, auf der Serviceebene, aber auch auf Ebene der Organisation bzw. der Gesellschaft erfolgreiche Initiativen zu ergreifen? Den empirischen Gegenstand unserer Untersuchung bilden gewerkschaftliche Beratungs- und Unterstützungseinrichtungen, die sich explizit an den spezifischen Interessen und Bedürfnissen von MigrantInnen orientieren. Die Untersuchung basiert auf Daten, die im Rahmen von rund 20 leitfadengestützten Interviews mit Beschäftigten von ÖGB und AK, die entweder als muttersprachliche BeraterInnen (BR) oder in leitender Funktion (LF) in solchen Einrichtungen tätig waren, erhoben wurden (vgl. Griesser und Sauer 2014). Im Zentrum der zwischen April 2013 und August 2014 in fünf österreichischen Bundesländern (Burgenland, Oberösterreich, Tirol, Vorarlberg, Wien) geführten und in der Folge inhaltsanalytisch ausgewerteten ExpertInneninterviews (vgl. etwa Gläser und Laudel 2010, S. 43 ff.; Bogner et al. 2014, S. 71 ff.) standen die Arbeits- und Funktionsweisen der erwähnten Einrichtungen sowie die Bedingungen und Kriterien des Gelingens der von diesen geleisteten Beratungs- und Unterstützungsarbeit.

Im Folgenden stellen wir kurz die MigrantInnen adressierenden Angebote vor, nicht zuletzt deshalb, weil diese in der Migrations- und Gewerkschaftsforschung in Österreich bislang gleichermaßen unterbelichtet blieben. 


\section{Gewerkschaftliche Beratungs- und Unterstützungsangebote: der empirische Gegenstand}

In der akademischen Auseinandersetzung mit dem Thema Gewerkschaften und Migration fanden die von österreichischen ArbeitnehmerInnenorganisationen etablierten Beratungs- und Unterstützungseinrichtungen lange Zeit allenfalls am Rande Beachtung (vgl. etwa Bauböck 1990; Gächter 2000). Erst in der jüngsten Vergangenheit wurden einige dieser Einrichtungen zum Gegenstand von sozialwissenschaftlichen Untersuchungen (vgl. etwa Hammer 2010a, 2010b) und - begriffen als Beispiele guter Praxis - von politischen Empfehlungen (vgl. etwa Biffl 2011, S. 133; Biffl et al. 2011, S. 28).

Eines der historisch gesehen ersten Serviceangebote, das explizit auf die spezifischen Bedürfnisse und Interessen von MigrantInnen fokussierte, waren Dolmetschund Übersetzungsleistungen für Türkisch/Kurdisch sowie Bosnisch/Kroatisch/ Serbisch (BKS) bzw. - wie es damals hieß - Serbokroatisch. Obschon im internationalen Vergleich von eher randständiger Bedeutung (vgl. Penninx und Roosblad 2000), wurden solche Angebote ab Mitte der 1960er-Jahre in mehreren österreichischen Bundesländern vom ÖGB und/oder der Arbeiterkammer etabliert. Im Laufe der Jahre und Jahrzehnte um-, zum Teil aus-, mitunter aber auch wieder zurückgebaut, entstanden daraus unterschiedlich konzipierte Modelle der muttersprachlichen MigrantInnenberatung, wie sie aktuell etwa beim ÖGB in Wien, Vorarlberg, Oberösterreich, Tirol und im Burgenland existieren (vgl. Griesser und Sauer 2014). ${ }^{7}$

In den vergangenen Jahren initiierte der ÖGB zudem auf Bundes- bzw. Länderebene mehrere Kooperationsprojekte (KP) mit ungarischen, slowakischen und tschechischen Gewerkschaften, die ebenfalls muttersprachliche Beratungs- und Unterstützungsangebote für MigrantInnen bzw. PendlerInnen umfassten. Damit reagierten die ArbeitnehmerInnenorganisationen auf die neuen Herausforderungen, wie sie mit der angesprochenen Transnationalisierung des Arbeitsmarkts und den damit verbundenen Migrationsbewegungen einhergingen (vgl. etwa Krings 2009, S. 57). Finanziert waren diese Einrichtungen zumeist über die Strukturfonds der Europäischen Union, allen voran dem Europäischen Fonds für Regionalentwicklung (EFRE), und formal basierten sie häufig auf den über den Europäischen Gewerkschaftsbund (EGB) organisierten Strukturen der Interregionalen Gewerkschaftsräte (IGRs) (vgl. Noack 2001; Hammer 2010b). ${ }^{8}$

\footnotetext{
7 In einigen Bundesländern werden diese Angebote vom ÖGB alleine (z. B. in Vorarlberg) bzw. in Kooperation mit der AK (z. B. in Oberösterreich) oder mit unabhängigen Beratungseinrichtungen (z. B. in Tirol) betrieben. In anderen Bundesländern finden sich parallel zu den Angeboten des ÖGB auch separate Angebote der AK (z. B. in Wien). Zu den ÖGB-Einrichtungen vgl. die Website: www.mitgliederservice. at/cms/S06/S06_50.5/beratungen (31.07.2016).

8 Konkret geht es im Folgenden um die beiden vom ÖGB Burgenland in Kooperation mit ungarischen Gewerkschaften entwickelten Projekte IGR Burgenland - Westungarn und Zukunft im GrenzRaum bzw. um die auf Bundesebene in Kooperation mit slowakischen Gewerkschaften entwickelten Projekte ZUWINBAT und Arbeitsmarkt+ sowie die in Kooperation mit tschechischen Gewerkschaften entwickelten Projekte $Z U$ WINS und ZUWINS+. Während die Beratung in tschechischer bzw. slowakischer Sprache nach Ablauf der Projekte wieder eingestellt wurde, gelang es dem ÖGB Burgenland, die Beratung in ungarischer Sprache im Rahmen des Projekts MIG - Mehrsprachige Beratung vorläufig zu verstetigen. Beim ÖGB Wien star-
} 
Die mit der Etablierung solcher Unterstützungs- und Beratungsangebote verbundene Motivation wurde im ÖGB-Grundsatzprogramm 2009 wie folgt gefasst:

Der ÖGB fordert [...], ArbeitsmigrantInnen durch Angebote wie Rechtsberatung und Rechtsschutz zu unterstützen. Der ÖGB sieht in der WanderarbeitnehmerInnenschaft eine wichtige Zielgruppe, deren gewerkschaftliche Organisierung einen wichtigen Beitrag zur Stabilisierung des Arbeitsmarkts und zur Sanierung prekärer Arbeitsverhältnisse leisten kann (ÖGB 2009, S. 58 f.).

Bei diesen gewerkschaftlichen Angeboten ist zu bedenken, dass (arbeits- und sozialrechtliche) Beratungstätigkeiten grundsätzlich nicht in den Kernaufgabenbereich des ÖGB, sondern - entsprechend einer komplexen Arbeitsteilung zwischen Betriebsräten, Gewerkschaften und Arbeiterkammern als den zentralen Institutionen der österreichischen ArbeitnehmerInnenvertretung (vgl. Karlhofer 2006) - in jenen der AK fällt. Verglichen mit den jährlich rd. zwei Millionen Beratungskontakten der AK nehmen sich etwa die rd. 2300 persönlichen Beratungen in Türkisch/ Kurdisch sowie die rd. 2000 persönlichen Beratungen in BKS, die laut Bericht für den 18. Bundeskongress im Berichtzeitraum 2009-2013 vom ÖGB-Servicecenter in Wien durchgeführt wurden (vgl. ÖGB 2013, S. 18), so auch bescheiden aus. Nicht außer Acht gelassen werden darf jedoch, dass das Aufgabenprofil der ÖGBBeraterInnen stets viel breiter angelegt war und eine Reihe anderer Aktivitäten von aufsuchender Arbeit in den Betrieben bis hin zu gewerkschaftlichen Organisierungstätigkeiten - umfasste.

In der Literatur wurde das mit diesen Einrichtungen verbundene Verhältnis von ÖGB und AK zu MigrantInnen(-Organisationen) vor allem in historischer Perspektive - aufgrund ihrer ambivalenten Positionierung zu Fragen der Migrationspolitik bzw. der gewerkschaftlichen Inklusion (vgl. Griesser und Sauer 2015) ${ }^{9}$ - verschiedentlich als ,klientelistisch“ und ,paternalistisch“ charakterisiert (vgl. etwa Bratić 2004, S. 66 f.; Waldrauch und Sohler 2004, S. 646; Sohler 2007, S. 384). Auch unter den BeraterInnen selbst kritisieren einige das zugrundeliegende Selbstverständnis der österreichischen ArbeitnehmerInnenorganisationen als paternalistische ,Schutzmacht für Ausländer“ (Interview ÖGB_BR_03): „Wir sind für euch da. Ihr müsst uns bei Wahlen eure Stimmen geben und wir machen's für euch. Dolmetscher? Bitteschön! Oder: Habt ihr einen Fußballverein? Kriegt ihr Uniformen, Mieten, Un-

tete im Frühjahr 2016 zudem ein neues Projekt mit arbeits- und sozialrechtlichen Beratungsangeboten in bulgarischer und rumänischer Sprache.

9 Hinsichtlich der Frage gewerkschaftlicher Inklusion war von zentraler Bedeutung, dass Personen ohne österreichische (bzw. später ohne EU/EWR-)Staatsbürgerschaft bis ins Jahr 2006 das passive Wahlrecht bei Arbeiterkammer- und Betriebsratswahlen vorenthalten blieb (vgl. Kreisky 2013, S. 174 f.; Griesser und Sauer 2015, S. 60 ff.). Da die Betriebsratsarbeit für Gewerkschaften in Österreich einen zentralen Mechanismus der Rekrutierung von FunktionärInnen und Hauptamtlichen darstellt, blieben die Möglichkeiten gewerkschaftlicher Teilhabe und Repräsentation für MigrantInnen aufgrund dieses Ausschlusses bis vor kurzem weitgehend auf die einfache Mitgliedschaft beschränkt (vgl. Gächter 2000, S. 77 ff.; Bauböck 1990, S. 49 f.). Gemäß einer Studie aus dem Jahr 1983 waren zu diesem Zeitpunkt nichtsdestotrotz rund $56 \%$ der ArbeiterInnen türkischer bzw. jugoslawischer Herkunft in Österreich gewerkschaftlich organisiert, was in etwa dem Organisationsgrad des ÖGB im Allgemeinen entsprach (vgl. Wimmer 1986, S. 263). Da der ÖGB weder die Staatsangehörigkeit noch den Migrationshintergrund seiner Mitglieder erfasst, gibt es hinsichtlich des gewerkschaftlichen Organisationsgrads von MigrantInnen und von dessen Entwicklung über die Zeit ansonsten jedoch kaum zuverlässige (Längsschnitt-)Daten. 
terstützung ...“(ebd.). Zudem sehen manche in den Angeboten deshalb reine „Alibistellen“ (Interview ÖGB_BR_02) bzw. „Alibijobs“ (Interview AK_BR_02), weil die personellen Ressourcen vor allem in den ersten Jahrzehnten ihres Bestehens aufgrund der massiven Nachfrage völlig unzureichend gewesen seien. Zugleich wird jedoch darauf verwiesen, dass ÖGB und AK gerade in den 1970er- und 1980erJahren in mancher Hinsicht die einzigen Organisationen waren, von denen überhaupt finanzielle, informationelle oder andere Unterstützungsangebote kamen, und dass die Zusammenarbeit in dieser Hinsicht auch ,ganz gut funktioniert[e]“" (Bratić 2000, S. 13; vgl. auch Bratić 2003, S. 399 ff.): „Ich muss ehrlich sagen, es gab damals keine anderen Ansprechpartner wegen Unterstützung, Hilfe, Beratung oder Rechtsschutz ... Und die Leute sind auch Mitglieder geworden, da gab es keine Diskussion.“ (Interview ÖGB_BR_02)

Vor dem Hintergrund eines inkrementellen Wandels des Verhältnisses österreichischer ArbeitnehmerInnenorganisationen zum Thema Migration, wie er etwa in einer stärkeren Inklusion von MigrantInnen in gewerkschaftliche Strukturen oder in einer punktuellen Neupositionierung von AK und ÖGB zu migrationspolitischen Fragen zum Ausdruck kommt (vgl. Griesser und Sauer 2015), scheint sich in jüngster Zeit - wie wir im Folgenden darlegen - auch die Bedeutung solcher Serviceangebote gewandelt zu haben.

\section{Gewerkschaftliche Erneuerung: MigrantInnen gewinnen}

Bei ihrem Versuch, MigrantInnen mit der Absicht ihrer gewerkschaftlichen Organisierung als (neue) Zielgruppe zu adressieren, verfolgen die österreichischen ArbeitnehmerInnenorganisationen im Rahmen der Beratungs- und Unterstützungseinrichtungen bislang vorrangig eine Dienstleistungsstrategie. Wie die Interviews mit den GewerkschafterInnen verdeutlichten, lässt sich diese Strategie jedoch nicht auf einen simplen Service-Ansatz reduzieren. Ähnlich wie im Falle internationaler Beispiele guter Praxis (vgl. etwa Benz 2014, S. 208 f.; Fine und Holgate 2014, S. 146 f.) kam es auch in Österreich zu einer Erweiterung des gewerkschaftlichen Strategierepertoires, indem Elemente traditioneller Dienstleistungsansätze mit solchen innovativer Organisierungsansätze gekoppelt wurden (vgl. etwa Hammer 2010a, S. 34/42 f.). Unter anderem aufgrund dieses - in der Literatur als „Organizing by servicing“ (Greer et al. 2013, S. 12) bezeichneten - Modells scheint es sinnvoll, die auf der Serviceebene lokalisierten Maßnahmen im Kontext umfassenderer Versuche einer gewerkschaftlichen Erneuerung zu verorten. ${ }^{10}$

Die im Folgenden dargestellten Gütekriterien, die wir aus den ExpertInneninterviews extrahieren konnten, werden entlang dreier Achsen sortiert. Dabei bezieht sich die Darstellung auf zwei zentrale Referenzen (vgl. Griesser und Sauer 2014,

\footnotetext{
10 Das Verfolgen eines solchen „Organizing by servicing“-Ansatzes ist kein Alleinstellungsmerkmal österreichischer ArbeitnehmerInnenorganisationen (vgl. etwa für Großbritannien Hardy et al. 2012, S. 360; Mustchin 2012, S. 965). Anstelle des in der Revitalisierungs-Literatur häufig bemühten ,shift in goals from servicing to organizing“ (Voss und Sherman 2000, S. 316) wird im Rahmen dieses Ansatzes eine (selektive) Integration der beiden Ziele versucht, wobei hier wesentliche inhaltliche und strukturelle Dimensionen des Organizing-Ansatzes häufig ausgespart bleiben (vgl. etwa Schröder 2015, S. 31/233 ff.).
} 
S. 64 ff.): Einerseits geht es um drei für das Verhältnis von Gewerkschaften zu Fragen der Migration verbindliche Dilemmata, die Rinus Penninx und Judith Roosblad (2000) in ihrer Studie Trade Unions, Immigration, and Immigrants in Europe herausgearbeitet haben. Das erste Dilemma betrifft die Entscheidung zwischen Kooperation oder Widerstand im Zusammenhang mit der Anwerbung bzw. Beschäftigung von MigrantInnen; das zweite bezieht sich auf die Frage ihrer (vollen) Inklusion in bzw. ihrer (partiellen) Exklusion aus gewerkschaftlichen Strukturen; und das dritte zielt auf die Wahl zwischen der Anwendung gleicher und der Entwicklung spezieller Maßnahmen für MigrantInnen. Andererseits greift die Darstellung einen Vorschlag von Petra Wlecklik (2013) auf, der mit Blick auf ein Fallbeispiel aus Deutschland entwickelt wurde. Dieser ordnet die gewerkschaftlichen Aktivitäten im Rahmen

Tab. 1 Gütekriterien einer gewerkschaftlichen Adressierung von MigrantInnen in Österreich. (Quelle: Eigene Erhebungen)

\begin{tabular}{ll}
\hline Ebenen und Dilemmata & Gütekriterien \\
\hline (1) Serviceebene: Entwicklung spezi- & (a) Beschäftigung eines durch Sprach- und ,interkulturelle \\
eller statt Anwendung gleicher Maß- & Kompetenzen“, rechtliche und v. a. praktische Expertise, \\
nahmen für MigrantInnen & „Parteilichkeit“ sowie Vernetzung in der Community sich \\
& auszeichnenden Personals; \\
& (b) Nutzung von Mundpropaganda sowie (flankierend) von \\
& Presse- und Öffentlichkeitsarbeit, Websites, Social Media \\
& usw. als Kanäle zwecks Verbreitung von Informationen zu \\
& den Angeboten; \\
& (c) Hinsichtlich der Rahmenbedingungen Akzentuierung von \\
& Vielfalt bei den Beratungsmedien und -gegenständen sowie \\
& von Flexibilität bei den Beratungsorten und -zeiten; \\
& (d) Ergänzung der Beratungstätigkeit durch zusätzliche An- \\
& bote wie Interventionen und Begleitungen, Vermittlung von \\
& Rechtsschutz, Betriebs- und aufsuchende Arbeit, Bildungsar- \\
& beit usw.
\end{tabular}

(2) Ebene der Organisation: (Volle) Inklusion in statt (partielle) Exklusion aus gewerkschaftlichen Strukturen
(3) Ebene der Gesellschaft: Kooperation statt Widerstand im Zusammenhang mit der Anwerbung/ Unterstützung von MigrantInnen (a) Bündelung migrationspolitischer Verantwortlichkeiten und Kompetenzen in einer Einrichtung innerhalb der Organisation;

(b) Gewährleistung der Selbstorganisation und -vertretung der Zielgruppe über eine eigene Plattform innerhalb der Organisation;

(c) Berücksichtigung der Bedürfnisse und Interessen von MigrantInnen in allen Bereichen durch Verfolgung einer Diversitäts-Strategie;

(d) Stärkung der Vernetzung ,innerhalb des Hauses“ als auch jenseits davon (z. B. im transnationalen Rahmen);

(e) Verbesserung der Repräsentation von MigrantInnen auf Ebene der Hauptamtlichen und FunktionärInnen

(a) Formulierung klarer Standpunkte zu migrations- und integrationspolitischen Fragen;

(b) Generierung von Wissen und Entwicklung von Expertise zu solchen Themen;

(c) Offensive Kommunikation der formulierten Standpunkte über unterschiedliche Medien und Formate;

(d) Stärkung der Vernetzung nach außen im Sinne des Coalition-Building (mit migrantischen Selbstorganisationen, NGOs usw.) 
eines topologischen Modells drei Ebenen - der Serviceebene, der Ebene der Organisation bzw. der Ebene der Gesellschaft - zu, auf denen auch die Dilemmata von Penninx und Roosblad lokalisiert werden können (vgl. dazu Tab. 1). Von zentraler Bedeutung wird aufgrund der dargelegten Dominanz der Dienstleistungsstrategie im Folgenden die erstgenannte Ebene sein.

\subsection{Faktoren auf der Serviceebene}

Die Gütekriterien einer solidarischen Beratungs- und Unterstützungsarbeit auf der Serviceebene umfassen - wegen der häufig prekären Situation und der daraus resultierenden Vulnerabilität der Zielgruppe - laut den interviewten GewerkschafterInnen möglichst niederschwellige Angebote für MigrantInnen.

In Bezug auf (a) das Personal ergeben sich daraus Gütekriterien wie Sprachund ,interkulturelle Kompetenzen“, rechtliche und vor allem praktische Expertise, „Parteilichkeit“ sowie Vernetzung in der Community: Während die Bedeutung ,interkultureller Kompetenzen“ bzw. eigener Migrationserfahrungen aufseiten der BeraterInnen in den Interviews nur vereinzelt angesprochen wurde (vgl. u. a. Interviews ÖGB_BR_04, AK_BR_03), bestand in Bezug auf die Relevanz entsprechender Sprachkompetenzen weitgehende Einigkeit (vgl. u. a. Interviews ÖGB_BR_02, KP_BR_01). Was die erforderliche Expertise anbelangt, blieb der inhaltliche Schwerpunkt - für gewerkschaftliche Einrichtungen wenig überraschend - über die Jahrzehnte hinweg unverändert im Bereich des Arbeits- und Sozialrechts. Ebenso unverändert blieb in der Regel jedoch für die BeraterInnen die Notwendigkeit, auch in anderen Feldern über rechtliche (z. B. zum Fremdenrecht) bzw. praktische Expertise (z. B. zu relevanten Behörden) zu verfügen (vgl. u. a. Interviews ÖGB_BR_01, KP_LF_03). Vor allem seitens der ersten Generation von BeraterInnen, die zwischenzeitlich überwiegend bereits pensioniert sind, wurde zudem die Bedeutung einer ,,parteilichen“ Beratung im Interesse der Ratsuchenden akzentuiert, also - wie ein oberösterreichischer Gewerkschafter es formulierte „dass man dieses Vertrauen hat: Der ist auf unsrer Seite“ (Interview ÖGB_BR_03). Vornehmlich von Angehörigen dieser Generation wurde darüber hinaus auch die Verankerung und der Status der BeraterInnen in der jeweiligen Community als ein relevanter Faktor betrachtet (vgl. u. a. Interviews ÖGB_BR_05, AK_BR_01).

Hinsichtlich (b) der Kanäle, über die Informationen zu den Angeboten verbreitet werden, wurde in den Interviews auf einen zentralen und eine Reihe ergänzender Kanäle verwiesen: In keinem anderen Punkt bestand dabei - über alle historischen und geografischen Distanzen zwischen den einzelnen Einrichtungen hinweg - größere Einigkeit unter den BeraterInnen als im Hinblick auf die Bedeutung von Mundpropaganda als zentralem Kanal für die Verbreitung von Informationen zu den Angeboten (vgl. u. a. Interviews KP_BR_01, AK_LF_01). Weniger Einigkeit gab es im Hinblick auf die Frage der Presse- und Öffentlichkeitsarbeit: Während diese seitens einzelner Einrichtungen mit Blick auf vor allem Regional- und Lokalmedien in anlassbezogener Form betrieben wurde (vgl. u. a. Interviews ÖGB_LF_01, KP_LF_03), fokussierten andere auf gewerkschaftliche bzw. auf Medien der relevanten Communitys (vgl. u. a. Interviews ÖGB_BR_01, ÖGB_BR_04) oder verzichteten auf entsprechende Aktivitäten (vgl. u. a. Interviews ÖGB_LF_02, AK_LF_01). 
Hinzu kamen von den Einrichtungen selbst produzierte Drucksorten (z. B. Plakate, Folder), die in Betrieben, Community- und anderen Einrichtungen verteilt wurden (vgl. u. a. Interviews ÖGB_LF_01, KP_BR_02). Vor allem in jüngster Zeit gewannen zudem E-Mail- und diverse andere Social-Media-Kanäle sowie der Webauftritt der verschiedenen Einrichtungen als Informationsmedien an Bedeutung (vgl. u. a. Interviews ÖGB_BR_04, KP_LF_03).

In Bezug auf (c) die Rahmenbedingungen wurde in medialer sowie inhaltlicher Hinsicht Vielfalt als zentrales Gütekriterium bemüht und in geografischer sowie zeitlicher Hinsicht Flexibilität: Was die Beratungs- und Informationsmedien anbelangt, wurde in den Interviews die Bedeutung vielfältiger Angebote betont. Lief dies in den ersten Jahrzehnten vor allem darauf hinaus, neben der persönlichen auch telefonische Beratung anzubieten und Informationen zudem etwa über Broschüren zu verbreiten (vgl. u. a. Interviews ÖGB_BR_02, ÖGB_BR_05), so spielt neuerdings E-Mail als Beratungsmedium eine zunehmend wichtigere Rolle (vgl. u. a. Interviews ÖGB_BR_04, KP_BR_01). Trotz des bereits angesprochenen Fokus auf arbeits- und sozialrechtliche Themen war Vielfalt darüber hinaus auch hinsichtlich der im Rahmen der Beratung abzudeckenden Rechts-Materien von zentraler Bedeutung. Als „Mädchen für alles“ (Interview ÖGB_BR_02) informierten die BeraterInnen deshalb auch in formeller bzw. informeller Form zu zahlreichen weiteren Themen (z. B. Steuern, Aufenthalt, Familie) (vgl. u. a. Interviews KP_BR_02, AK_BR_01). Vor allem während der ersten Jahrzehnte ihres Bestehens war es zudem üblich, dass die muttersprachliche Beratung nicht bloß in den Gewerkschaftszentralen, sondern auch in den Bezirks- bzw. Regionalsekretariaten des ÖGB oder bei den Teilgewerkschaften angeboten wurde (vgl. u. a. Interviews ÖGB_BR_03, ÖGB_BR_05). Das galt in vergleichbarer Form für die internationalen Kooperationsprojekte, die jenseits ihrer Zentralen stets auch über ein Netz an Außenstellen verfügten (vgl. u. a. Interviews KP_BR_01, KP_LF_02). Darüber sowie durch das gezielte Aufsuchen von Betrieben bzw. anderen einschlägigen Orten (z. B. Grenzübergängen) sollte es gelingen, durch geografische Flexibilität im Sinne der angesprochenen Niederschwelligkeit ,näher an die Leute zu kommen“ (Interview KP_LF_03). Aus ähnlichen Gründen versuchte man vielerorts lange Zeit, ,, solche bürokratischen Sachen“ (Interview ÖGB_BR_03) wie fixe Beratungszeiten oder obligatorische Voranmeldungen zu vermeiden. Zwischenzeitlich ist letzteres zwar in den meisten Einrichtungen Usus; nichtsdestotrotz ist man zumeist auch weiterhin um zeitliche Flexibilität bemüht (vgl. u. a. Interviews ÖGB_BR_01, ÖGB_LF_01).

Bei den einzelnen Projekten von unterschiedlicher Bedeutung, im Hinblick auf die Frage gewerkschaftlicher Organisierung jedoch zentral, waren bzw. sind schließlich (d) Angebote, welche die Beratungstätigkeit im engeren Sinn ergänzen: Neben (telefonischen) Interventionen bei und zum Teil auch persönlichen Begleitungen zu ArbeitgeberInnen und Behörden umfasste dies vor allem die Weitervermittlung in den Rechtsschutz der zuständigen Teilgewerkschaft bzw. der Arbeiterkammern. Unter anderem in Abhängigkeit von den personellen Ressourcen der jeweiligen Einrichtung kam mitunter Informations- und Bildungsarbeit oder auch Betriebs- und aufsuchende Arbeit hinzu (vgl. u. a. Interviews ÖGB_BR_01, KP_LF_02).

Neben den dargestellten Faktoren auf der Serviceebene wurden in den Interviews von den GewerkschafterInnen aber auch flankierende Maßnahmen auf Ebene der 
Organisation bzw. der Gesellschaft angesprochen, worauf im Folgenden kurz eingegangen werden soll.

\subsection{Faktoren auf der Ebene der Organisation}

Auf der Ebene der Organisation wurde von vielen Interviewten unter anderem mit Verweis auf entsprechende Wünsche seitens migrantischer BetriebsrätInnen (a) die Schaffung einer Einrichtung innerhalb der Organisation angeregt, in der migrationsund integrationspolitische Verantwortlichkeiten und Kompetenzen gebündelt werden sollen (vgl. u. a. Interview ÖGB_BR_01, AK_LF_02). Dies sei, wie ein oberösterreichischer Gewerkschafter betont, in der Vergangenheit auch immer wieder Gegenstand von Forderungen gewesen:

Wir haben immer wieder von der Zentrale verlangt, dass es in der Organisationsform des ÖGB ein Migrationsreferat oder -sektion oder -abteilung geben soll [...]. Das war immer eine Forderung von uns in Kongressen [...]; hat's aber nie gegeben. Ich werd's wahrscheinlich auch nicht erleben (Interview ÖGB_BR_03).

Daneben plädierten mehrere Interviewte für (b) die Schaffung einer Plattform innerhalb der Organisation, über die im gewerkschaftlichen Rahmen die Selbstorganisation und -vertretung der Zielgruppe ermöglicht werden soll, wofür es in Österreich auch bereits einige Beispiele guter Praxis gebe (z. B. das 2009 beim ÖGB Oberösterreich eingerichtete Kompetenzzentrum Migration) (vgl. u. a. Interviews ÖGB_BR_03, ÖGB_BR_04).

Ein weiterer in den Interviews wiederholt angesprochener Aspekt war (c) die Verfolgung einer Diversitäts-Strategie in der gesamten Organisation zwecks Berücksichtigung der besonderen Bedürfnisse und Interessen von MigrantInnen im Rahmen aller internen und Außenbeziehungen (vgl. u. a. Interviews AK_LF_01, AK_LF_02). In gewerkschaftlichen Kreisen wurde hier vor allem die Bedeutung der Bildungsarbeit akzentuiert, um die ,interkulturelle Kompetenz“ von Hauptamtlichen und FunktionärInnen ebenso wie von BetriebsrätInnen - auch jenseits der mit dem Thema befassten Kreise - zu vertiefen (vgl. u. a. Interviews ÖGB_BR_01, ÖGB_BR_03).

Darüber hinaus sprachen viele Interviewte (d) die Stärkung der Vernetzung als wichtigen Aspekt an - und zwar sowohl ,innerhalb des Hauses“ (zwischen Selbstvertretungs-, politischen und Serviceabteilungen) als auch jenseits davon (innerhalb des ÖGB bzw. der AK; zwischen ÖGB, AK und Teilgewerkschaften) (vgl. u. a. Interviews KP_BR_02, AK_BR_03). Besonders häufig wurde dabei - zum Teil unter Verweis auf (historische) Erfahrungen in diesem Feld - der Wunsch nach Möglichkeiten des Austauschs und der Vernetzung unter den BeraterInnen selbst artikuliert (vgl. u. a. Interviews ÖGB_BR_02, AK_BR_01). Vor allem die mit den grenzüberschreitenden Kooperationsprojekten verbundenen GewerkschafterInnen akzentuierten zudem die Bedeutung des transnationalen Wissenstransfers im gewerkschaftlichen Rahmen (vgl. u. a. Interviews KP_LF_01, KP_LF_03).

Als kontroversester Punkt wurde schließlich in nahezu allen Interviews (e) auf die Frage einer verstärkten Repräsentation von MigrantInnen auf Ebene der Hauptamt- 
lichen und FunktionärInnen eingegangen. Im Hinblick auf das Ziel bestand dabei zwar weitgehende Einigkeit (vgl. u. a. Interviews ÖGB_BR_03, ÖGB_LF_01). Was die dafür zum Einsatz zu bringenden Mittel anbelangt - konkret etwa Maßnahmen wie Affirmative Action in der Personalpolitik oder Quotenregelungen bei der Vergabe von Funktionen -, dominierte jedoch Ambivalenz (vgl. u. a. Interviews ÖGB_BR_01, ÖGB_BR_04).

\subsection{Faktoren auf der Ebene der Gesellschaft}

Auf der Ebene der Gesellschaft wurde unter anderem (a) auf die Notwendigkeit der Entwicklung klarer Standpunkte zu migrations- und integrationspolitischen Fragen verwiesen. In der Perspektive mancher InterviewpartnerInnen soll dies entweder im Rahmen umfassender Positions- bzw. Standpunktpapiere passieren (vgl. u. a. Interviews ÖGB_LF_03, AK_LF_03); oder auch in Bezug auf konkrete Themen wie etwa die Übergangsfristen für die ArbeitnehmerInnenfreizügigkeit von BürgerInnen der neuen EU-Mitgliedstaaten (vgl. u. a. Interview KP_LF_03, ÖGB_LF_01).

Um eine solche Positionierung vor dem Hintergrund lückenhafter Wissensbestände zu ermöglichen, sei es laut mehreren Interviewten zudem von zentraler Bedeutung, (b) Wissen zu generieren und Expertise zu entwickeln. Verwiesen wurde dabei beispielsweise auf die Erarbeitung wissenschaftlicher Studien zu migrantischen BetriebsrätInnen (vgl. Interview ÖGB_BR_01); oder allgemein darauf, dass „Felder, die für Integration wichtig sind, studienmäßig erfasst werden [...] mit der Option, politikmächtiges Handeln daraus ableiten zu können“ (Interview AK_LF_02).

Einmal entwickelt, sollen die angesprochenen Positionen, wie manche InterviewpartnerInnen betonten, auch (c) offensiv nach außen kommuniziert werden. Dafür infrage kämen Kampagnen, die verschiedene AkteurInnen einbinden und adressieren (vgl. u. a. Interviews ÖGB_LF_03, AK_LF_02), oder auch klassische Pressearbeit, die - wie eine Tiroler Gewerkschaftssekretärin meinte - auch als vertrauensbildende Maßnahme gegenüber der Zielgruppe wirke:

Das ist ganz wichtig [...]. Wir machen auch immer wieder diesbezüglich Aussendungen, Pressearbeit. Jetzt zum Beispiel zu ,50 Jahre Gastarbeiter“ haben wir eine Presseaussendung geschrieben. [...] Und ja, das ist für die schon von Relevanz: Ich merk schon, dass in letzter Zeit das Vertrauen uns gegenüber wesentlich höher geworden ist (Interview ÖGB_LF_01).

Schließlich sahen viele der interviewten GewerkschafterInnen (d) die Vernetzung nach außen im Sinne des Coalition-Building mit migrantischen Selbstorganisationen und Community-Einrichtungen sowie mit NGOs und unabhängigen Beratungseinrichtungen als zentral an. Im Beratungsalltag funktioniere dies vor allem im Bereich der etablierten Einrichtungen von ÖGB und/oder AK vielerorts gut (vgl. u. a. Interviews ÖGB_LF_01; AK_BR_02), was zum Teil auch für die internationalen Kooperationsprojekte gelte (vgl. u. a. Interviews KP_BR_02, KP_LF_03). Jenseits davon, also beispielsweise im Rahmen politischer Kampagnen, passiere dies bislang aber nur punktuell bzw. anlassbezogen (vgl. u. a. Interview ÖGB_LF_03, AK_LF_02). 


\section{Schlussfolgerungen}

Auf der Basis der in Tab. 1 in komprimierter Form dargestellten Ergebnisse unserer Untersuchung zu Gütekriterien gewerkschaftlicher Beratungs- und Unterstützungsarbeit für MigrantInnen lassen sich im Anschluss an Penninx und Roosblad (2000) bzw. Wlecklik (2013) mit Blick auf die forschungsleitende Frage folgende Schlussfolgerungen ziehen: Was die Serviceebene anbelangt, ist mit Wlecklik gesprochen eine ,zielgruppenspezifische Ansprache der Beschäftigten mit Migrationshintergrund“ anzustreben. Mit Blick auf das dritte von Penninx und Roosblad formulierte Dilemma setzt dies voraus, dass aufgrund der besonderen Bedürfnisse und Interessen von MigrantInnen die Entwicklung spezieller Angebote durch Gewerkschaften erforderlich ist.

Wie aus den Interviews hervorging, ist der ausschließliche Fokus auf die Serviceebene für eine erfolgreiche Adressierung von MigrantInnen als (neue) Zielgruppe jedoch unzureichend. In der Literatur zum Thema wird in diesem Zusammenhang entsprechend auf die Notwendigkeit eines „,comprehensive effort“ (Milkman und Wong 2001, S. 128) verwiesen. Laut Milkman und Wong bedürfen solche Angebote nämlich der Ergänzung durch Veränderungen auf Ebene der Organisation (Stichwort: „effectively incorporating immigrants into leadership at all levels“) bzw. auf Ebene der Gesellschaft (Stichwort: ,signal its support of immigrant workers on a broader level“") (vgl. ebd.; vgl. ähnlich Fitzgerald und Hardy 2010, S. 140 ff.).

Entsprechend ist flankierend zu den dargestellten Maßnahmen auf der Serviceebene auf Ebene der Organisation das zu entwickeln, was Wlecklik eine ,interkulturell ausgerichtete Personal- und Organisationspolitik“" nennt. Mit Blick auf das zweite von Penninx und Roosblad dargelegte Dilemma impliziert dies, dass das erstrebenswerte Ziel einer vollen Inklusion von MigrantInnen nicht bloß die Zuerkennung gleicher Rechte, sondern eine Transformation der gesamten Organisation(skultur) erfordert (vgl. auch Artus 2011, S. 227; Adler und Cornfield 2014, S. 42 ff.). Auf Ebene der Gesellschaft schließlich geht es im Anschluss an Wlecklik um eine „Themensetzung für MultiplikatorInnen in Wirtschaft, Politik und Gesellschaft“. Im Hinblick auf das erste von Penninx und Roosblad formulierte Dilemma setzt dies eine Neupositionierung von Gewerkschaften im Sinne der Verschiebung des Akzents von einer vornehmlich restriktiv-ausschließenden zu einer stärker solidarischeinschließenden Haltung gegenüber MigrantInnen voraus (vgl. auch Alberti et al. 2014, S. 129; Schröder 2015, S. 16 ff.).

Letztlich weisen die im vorliegenden Artikel beispielhaft behandelten Beratungsund Unterstützungspraxen Wege in Richtung einer gewerkschaftlichen Erneuerung, die den Einsatz für die Stärkung der Organisationsmacht von MigrantInnen zu einer zentralen Grundlage der organisationseigenen Stärke von Gewerkschaften macht.

Open access funding provided by University of Vienna.

Open Access Dieser Artikel wird unter der Creative Commons Namensnennung 4.0 International Lizenz (http://creativecommons.org/licenses/by/4.0/deed.de) veröffentlicht, welche die Nutzung, Vervielfältigung, Bearbeitung, Verbreitung und Wiedergabe in jeglichem Medium und Format erlaubt, sofern Sie den/die ursprünglichen Autor(en) und die Quelle ordnungsgemäß nennen, einen Link zur Creative Commons Lizenz beifügen und angeben, ob Änderungen vorgenommen wurden. 


\section{Literatur}

Adler, Lee, Maite Tapia, und Lowell Turner. 2014. Mobilizing against inequality. Unions, workers, und the crisis of capitalism. Ithaca/London: Cornell University Press.

Adler, Lee, und Daniel B. Cornfield. 2014. The United States: tackling inequality in precarious times. In Mobilizing against Inequality, Hrsg. Lee Adler, Maite Tapia, und Lowell Turner, 35-51. Ithaca London: Cornell University Press.

Alberti, Gabriella, Jane Holgate, und Lowell Turner. 2014. Opportunity and choice for unions organizing immigrant workers. A comparison across countries and industries. In Mobilizing against Inequality, Hrsg. Lee Adler, Maite Tapia, und Lowell Turner, 109-130. Ithaca London: Cornell University Press.

Arbeitskreis Strategic Unionism. 2013. Jenaer Machtressourcenansatz 2.0. In Comeback der Gewerkschaften? Machtressourcen, innovative Praktiken, internationale Perspektiven, Hrsg. Stefan Schmalz, Klaus Dörre, 345-375. Frankfurt New York: Campus.

Artus, Ingrid. 2011. Gewerkschaftliche Interessenvertretung im Niedriglohnsektor und der Streik der französischen Travailleurs sans papiers. In Gewerkschaftliche Modernisierung, Hrsg. Thomas Haipeter, Klaus Dörre, 208-230. Wiesbaden: VS.

Bauböck, Rainer. 1990. Kein Kurswechsel des ÖGB in der Ausländerpolitik. Kurswechsel 1:48-56.

Behrens, Martin, Kerstin Hamann, und Richard W. Hurd. 2004. Conceptualizing labour union revitalization. In Varieties of unionism: strategies for union revitalization in a globalizing economy, Hrsg. Carola M. Frege, John Kelly, 11-29. New York: Oxford University Press.

Benz, Martina. 2014. Zwischen Migration und Arbeit: Worker Centers und die Organisierung prekär und informell Beschäftigter in den USA. Münster: Westfälisches Dampfboot.

Biffl, Gudrun. 2011. Gewerkschaften und Zuwanderung in Österreich: MigrantInnen als neue Zielgruppe? In Grundzüge des Managements von Migration und Integration, Hrsg. Gudrun Biffl, Nikolaus Dimmel, 129-139. Leobersdorf: omninum.

Biffl, Gudrun, Lea Rennert, und Petra Aigner. 2011. Migrant workers in Austria and Europe: challenges for industrial relations, in particular trade unions. Krems: Department for Migration and Globalisation.

Biffl, Gudrun. 2016. Migration and labour integration in Austria. SOPEMI report on labour migration Austria 2014-15. Krems: Department for Migration and Globalisation.

Blaschke, Sabine. 2002. Der ÖGB: Gewerkschaftliche Reorganisation und Erneuerung. Kurswechsel 2:89-101.

Blaschke, Sabine. 2007. Austria: corporatist unionism in crisis. In Trade Union revitalisation: trends and prospects in 34 countries, Hrsg. Craig Phelan, 245-257. Oxford: Peter Lang.

Bogner, Alexander, Beate Littig, und Wolfgang Menz. 2014. Interviews mit Experten. Eine praxisorientierte Einführung. Wiesbaden: VS.

Bratić, Ljubomir. 2000. Soziopolitische Organisationen der MigrantInnen in Österreich. Kurswechsel 1:6-20.

Bratić, Ljubomir. 2003. Soziopolitische Netzwerke der MigrantInnen aus der ehemaligen Sozialistischen Föderativen Republik Jugoslawien (SFRJ) in Österreich. In Österreichischer Migrations- und Integrationsbericht, Hrsg. Heinz Fassmann, Irene Stacher, 395-409. Klagenfurt Celovec: Drava.

Bratić, Ljubomir. 2004. Die Frage der Selbstorganisation. In Gastarbajteri. 40 Jahre Arbeitsmigration, Hrsg. Hakan Gürses, Cornelia Kogoj, und Sylvia Mattl, 61-68. Wien: Mandelbaum.

Brinkmann, Ulrich, Hae-Lin Choi, Richard Detje, Klaus Dörre, Hajo Holst, Serhat Karakayali, und Catharina Schmalstieg. 2008. Strategic Unionism: Aus der Krise zur Erneuerung? Wiesbaden: VS.

Choi, Hae-Lin. 2008. Re-Thinking Justice for Janitors - Strategische Erneuerung der amerikanischen Dienstleistungsgewerkschaft SEIU. WSI Mitteilungen 1:11-17.

Ebbinghaus, Bernhard. 2000. Austria. In Trade Unions in Western Europe since 1945, Hrsg. Bernhard Ebbinghaus, Jelle Visser, 77-91. Basingstoke Oxford: MacMillan Reference.

Janice, Fine, und Daniel J. Tichenor. 2012. An enduring dilemma: immigration and organized labor in western europe and the United States. In The Oxford Handbook of the politics of international migration, Hrsg. Marc R. Rosenblum, Daniel J. Tichenor, 532-572. Oxford New York: Oxford University Press.

Janice, Fine, und Jane Holgate. 2014. The countermovement needs a movement (and a Counterstrategy). In Mobilizing against Inequality, Hrsg. Lee Adler, Maite Tapia, und Lowell Turner, 131-150. Ithaca London: Cornell University Press.

Fitzgerald, Ian, und Jane Hardy. 2010. Thinking outside the box? Trade union organising strategies and Polish migrant workers in the United Kingdom. British Journal of Industrial Relations 48(1):131-150. doi:10.1111/j.1467-8543.2009.00761.x. 
Gächter, August. 2000. Protecting indigenous workers from immigrants: Austria. In Trade unions, immigration, and immigrants in Europe, 1960-1993, Hrsg. Rinus Penninx, Judith Roosblad, 65-89. New York Oxford: Berghahn Books.

Gläser, Jochen, und Grit Laudel. 2010. Experteninterviews und qualitative Inhaltsanalyse. Wiesbaden: VS.

Greer, Ian, Zinovijus Ciupijus, und Nathan Lillie. 2013. The European migrant workers union und the barriers to transnational industrial citizenship. European Journal of Industrial Relations 19(1):5-20. doi:10.1177/0959680112474748.

Griesser, Markus, und Birgit Sauer. 2014. MigrantInnen als Zielgruppe: Solidarische Beratungs- und Unterstützungsangebote von ArbeitnehmerInnenorganisationen in Österreich. Studie im Auftrag der AK Wien. Wien: Arbeiterkammer Wien.

Griesser, Markus, und Birgit Sauer. 2015. Zwischen „Konjunkturpuffer“ und „Tauschobjekt“. Gewerkschaftliche Perspektiven auf Migration im Österreich der Zweiten Republik. Kurswechsel 4:58-66.

Gstöttner-Hofer, Gerhard. 2005. Kampagnen als Erweiterung des gewerkschaftlichen Strategierepertoires in Österreich. Ursachen - Erfahrungen - Erkenntnisse. Wien: ÖGB.

Hammer, Nikolaus. 2010a. Regionen, Ebenen, Netzwerke: Interregionale Gewerkschaftskooperation im IGR Westpannonien. Wirtschaft und Gesellschaft 1:33-52.

Hammer, Nikolaus. 2010b. Cross-border cooperation under asymmetry: the case of an interregional trade union council. European Journal of Industrial Relations 16(4):351-367. doi:10.1177/ 0959680110384535.

Hardy, Jane, Line Eldring, und Thorsten Schulten. 2012. Trade union responses to migrant workers from the „new Europe“: a three sector comparison in Norway, Germany and the UK. European Journal of Industrial Relations 18(4):347-363. doi:10.1177/0959680112461464.

Hermann, Christoph, und Jörg Flecker. 2009. Betriebliche Interessenvertretung in Österreich: Wachsender Druck und zunehmende Lücken. In Die Dynamik des „österreichischen Modells “: Brüche und Kontinuitäten im Beschäftigungs- und Sozialsystem, Hrsg. Christoph Hermann, Roland Atzmüller, 93-110. Berlin: edition sigma.

Heery, Edmund, und Lee H. Adler. 2004. Organizing the Unorganized. In Varieties of unionism. Strategies for union revitalization in a globalizing economy, Hrsg. Carola Frege, John Kelly, 45-69. Oxford: Oxford University Press.

Holst, Hajo, Andreas Aust, und Susanne Pernicka. 2008. Kollektive Interessenvertretung im strategischen Dilemma - Atypische Beschäftigung und die ,dreifache Krise“ der Gewerkschaften. Zeitschrift für Soziologie 37(2):158-176.

Jandl, Michael, Christina Hollomey, und Anna Stepien. 2007. Migration and irregular work in Austria: results of a delphi-study. ILO-International Migration Papers. Genf: ILO.

Kalter, Frank. 2005. Ethnische Ungleichheit auf dem Arbeitsmarkt. In Arbeitsmarktsoziologie. Probleme, Theorien, empirische Befunde, Hrsg. Martin Abraham, Thomas Hinz, 303-332. Wiesbaden: VS.

Karlhofer, Ferdinand. 2006. Arbeitnehmerorganisationen. In Politik in Österreich. Das Handbuch, Hrsg. Herbert Dachs, et al., 462-479. Wien: Manz.

Karlhofer, Ferdinand. 2012. Österreich. Zwischen Korporatismus und Zivilgesellschaft. In Verbände und Verbandssysteme in Westeuropa, Hrsg. Werner Reutter, 521-549. Wiesbaden: VS.

Korpi, Walter. 1983. The Democratic Class Struggle. London Boston Melbourne Henley: Routledge.

Kreisky, Jan. 2013. Migration und Gewerkschaft. Skizze einer widerspruchsvollen und wechselhaften Geschichte. In Wissenschaft über Gewerkschaft, Hrsg. Brigitte Pellar, 155-187. Wien: ÖGB.

Krenn, Manfred, und Bettina Haidinger. 2009. Un(der)documented labour - characteristics, conditions and labour market impacts. FORBA Research Report 2/2009. Wien: FORBA.

Krenn, Manfred, Eva Saunders, und Bernhard Saupe. 2012. Prekäre Integration - die soziale Verwundbarkeit von MigrantInnen und ihre Integration durch Erwerbsarbeit. FORBA Research Report 4/2012. Wien: FORBA.

Krings, Torben. 2009. A race to the bottom? Trade unions, EU enlargement und the free movement of labour. European Journal of Industrial Relations 1:49-69. doi:10.1177/0959680108100165.

Meardi, Guglielmo, Antonio Martin, und Mariona Lozano Riera. 2012. Constructing uncertainty: Unions und migrant labour in construction in Spain and the UK. Journal of Industrial Relations 54(1):5-21. doi:10.1177/0022185611432388.

Meyer, Malte. 2002. Neuanfang in der Defensive. US-Gewerkschaften unter Handlungsdruck. Hamburg: VSA.

Milkman, Ruth, und Kent Wong. 2001. Organizing immigrant workers: case studies from southern california. In Rekindling the movement: labor's quest for relevance in the twenty-first century, Hrsg. Lowell Turner, Harry C. Katz, und Richard W. Hurd, 99-128. Ithaca: Cornell University Press. 
Mustchin, Stephen. 2012. Unions, learning, migrant workers and union revitalization in Britain. Work, Employment und Society 26(6):951-967. doi:10.1177/0950017012458022.

Noack, Jürgen. 2001. Interregional trade union councils in the run-up to eastern enlargement of the EU. In European trade union yearbook 2000, Hrsg. Emilio Gabaglio, Reiner Hoffmann, 337-354. Brüssel: ETUI.

OECD. 2016. Trade union density. http://stats.oecd.org/Index.aspx?QueryId=20167. Zugegriffen: 31. Juli 2016.

ÖGB. 2009. Grundsatzprogramm des ÖGB 2009-1013. Wien: ÖGB.

ÖGB. 2013. Bericht an den 18. Bundeskongress. Wien: ÖGB.

Olsen, Gregg M., und Julia S. O'Connor. 1998. Understanding the welfare state: power resources theory and its critics. In Power resources theory and the welfare state. A critical approach, Hrsg. Gregg M. Olsen, Julia S. O'Connor, 3-33. Toronto Buffalo London: University of Toronto Press.

Penninx, Rinus, und Judith Roosblad (Hrsg.). 2000. Trade Unions, Immigration, and Immigrants in Europe, 1960-1993. New York Oxford: Berghahn Books.

Pernicka, Susanne, und Sandra Stern. 2011. Von der Sozialpartnergewerkschaft zur Bewegungsorganisation? Mitgliedergewinnungsstrategien österreichischer Gewerkschaften. Österreichische Zeitschrift für Politikwissenschaft 4:335-355.

Phelan, Craig. 2007. Worldwide trends and prospects for trade union revitalisation. In Trade union revitalisation: trends and prospects in 34 countries, Hrsg. Craig Phelan, 245-257. Oxford: Peter Lang.

Riesenfelder, Andreas, Susanne Schelepa, und Petra Wetzel. 2011. Beschäftigungssituation von Personen mit Migrationshintergrund in Wien. Studie im Auftrag der AK Wien. Wien: AK Wien.

Schröder, Berit. 2015. Gewerkschaften und Wunderarbeit. Von Saisonarbeit, Werkverträgen und migrantischer Organisation in der Baubranche und im Grünen Bereich. Münster: Westfälisches Dampfboot.

Sengenberger, Werner. 1978. Arbeitsmarktstruktur: Ansätze zu einem Modell des segmentierten Arbeitsmarkts. Frankfurt am Main: Campus.

Silver, Beverly J. 2005. Forces of Labor. Arbeiterbewegungen und Globalisierung seit 1870. Berlin: Assoziation A.

Sohler, Karin. 2007. MigrantInnenorganisationen in Wien. In 2. Österreichischer Migrations- und Integrationsbericht 2001-2006, Hrsg. Heinz Fassmann, 377-391. Klagenfurt Celovec: Drava.

Tálos, Emmerich. 2008. Sozialpartnerschaft. Ein zentraler politischer Gestaltungsfaktor in der Zweiten Republik. Innsbruck: Studienverlag.

Traxler, Franz. 1993. Vom Nachfrage- zum Angebotskorporatismus? Sozialpartnerschaftliche Stabilitätsbedingungen im Wandel. In Sozialpartnerschaft: Kontinuität und Wandel eines Modells, Hrsg. Emmerich Tálos, 103-116. Wien: Verlag für Gesellschaftskritik.

Traxler, Franz. 1998. Austria - still the country of corporatism. In Changing industrial relations in europe, Hrsg. Anthony Ferner, Richard Hyman, 239-261. Oxford: Wiley-Blackwell.

Traxler, Franz, und Susanne Pernicka. 2007. The state of the unions: Austria. Journal of Labor Research 2:207-232.

Voss, Kim, und Rachel Sherman. 2000. Breaking the Iron Law of Oligarchy: Union Revitalization in the American Labor Movement. American Journal of Sociology 2:303-349.

Waldrauch, Harald, und Karin Sohler. 2004. Migrantenorganisationen in der Großstadt. Entstehung, Strukturen und Aktivitäten am Beispiel Wien. Frankfurt New York: Campus.

Wimmer, Hannes. 1986. Die Arbeitswelt der ausländischen Arbeitnehmer. In Ausländische Arbeitskräfte in Österreich, Hrsg. Hannes Wimmer, 241-280. Frankfurt New York: Campus.

Wlecklik, Petra. 2013. Partizipation von Migrant_innen und interkulturelle Öffnung am Beispiel der IG Metall. In: Heimatkunde. Migrationspolitisches Portal der Heinrich Böll Stiftung. https:// heimatkunde.boell.de/2013/09/10/partizipation-von-migrantinnen-und-interkulturelle-oeffnungam-beispiel-der-ig-metall. Zugegriffen: 31. Juli 2016.

Wright, Erik Olin. 2000. Working-Class Power, Capitalist-Class Interests and Class Compromise. American Journal of Sociology 105(4):957-1002. doi:10.1086/210397.

Markus Griesser war in den vergangenen Jahren an verschiedenen österreichischen Universitäten und Fachhochschulen tätig und ist zurzeit Projektmitarbeiter am Institut für Politikwissenschaft der Universität Wien. Seine Schwerpunkte in Forschung und Lehre liegen im Bereich Sozialstaatstheorie, Sozialhilfe- und Arbeitsmarktpolitik sowie Gewerkschafts- und soziale Bewegungen. 
Birgit Sauer ist seit 2001 Universitätsprofessorin am Institut für Politikwissenschaft der Universität Wien. Sie war Sprecherin des Graduiertenkollegs GiK (GenderInitiativKolleg) an der Universität Wien. Ihre Forschungsschwerpunkte sind Gender und Governance/Critical Governance-Studies, Vergleichende Geschlechterpolitikforschung, Demokratie und Differenz, Staats-, Demokratie- und Institutionentheorien sowie Emotionen und Politik. $\mathrm{Zu}$ diesen Themen hat sie umfangreich geforscht und publiziert (www.birgitsauer.org). 